\title{
ANÁliSE DO PROCESSO DE EXTRAÇÃO DE ÓLEO DE SOJA EM UM EXTRATOR DE ESTEIRA
}

\author{
A. TROMBETA ${ }^{1}$, L. V. DURANTE ${ }^{1}$, E. R. A. PRADO ${ }^{1}$, \\ P. R. PARAÍSO ${ }^{1}$ e L. M. M. JORGE ${ }^{1}$
}

${ }^{1}$ Universidade Estadual de Maringá, Departamento de Engenharia Química E-mail para contato: alessandrotrombeta@yahoo.com.br/lmmj@ deq.uem.br

\begin{abstract}
RESUMO - A indústria de óleo de soja constitui um importante ramo da agroindústria brasileira. A extração por solvente é a operação de transferência de massa mais utilizada para a obtenção do óleo. Há uma busca incessante por melhorias, visando a redução de custo e eliminação de desperdícios, apontando para a necessidade de um modelo matemático de um extrator industrial de esteira, devidamente validado, que represente o processo de extração, a fim viabilizar a análise e otimização do processo, procurando ampliar as vantagens econômicas e competitivas. Neste contexto, aplicou-se um modelo matemático baseado em balanços de massa, representado por um conjunto de equações diferenciais parciais. Este modelo foi resolvido numericamente por diferenças finitas no MAPLE® e foi validado frente a dados de uma planta industrial de extração de óleo de soja. Os resultados revelam que o modelo utilizado representou adequadamente o processo e pode ser empregado na simulação, análise e otimização de extratores industriais de óleo de soja.
\end{abstract}

\section{INTRODUÇÃO}

A soja é uma importante oleaginosa mundial e o Brasil se destaca como um dos maiores produtores mundiais. A Tabela 1 apresenta uma estimativa de produção para a safra 2013/2014 dos três maiores produtores mundiais, de acordo com o $7^{\circ}$ levantamento realizado pela USDA (United States Department of Agriculture). A estimativa mundial para a safra 2013/2014 é de 283,5 milhões de toneladas.

Tabela 1 - Produção Mundial de Soja, em Milhões de Toneladas

\begin{tabular}{|c|c|c|}
\hline \multirow{2}{*}{ Países } & \multicolumn{2}{|c|}{ Safras } \\
\cline { 2 - 3 } & $2012 / 2013$ & $2013 / 2014$ \\
\hline EUA & 82,6 & 88,7 \\
\hline Brasil & 82,0 & 88,0 \\
\hline Argentina & 49,3 & 53,5 \\
\hline
\end{tabular}

Demarco (2009) afirma que a indústria de azeites, principalmente vindos da soja, é milenária e permanece atuante, passando por avanços significativos nos últimos séculos. Para Boss (2000) a extração de óleos vegetais por solvente mostrou-se mais interessante que a extração utilizando prensas. Almeida et al. (2010) afirma que a extração sólido-líquido com 
solvente hexano é o processo mais usado nas indústrias de todo o mundo e que os extratores mais utilizados são os do tipo célula rotativa (Rotocell) e do tipo esteira.

Segundo Rataus (2000) em um processo industrial também é importante o conhecimento da matéria que será submetida ao processo de extração. A soja laminada possui três características importantes: o espaço entre as superfícies externas das lâminas, os capilares microscópicos que constituem a matéria porosa e os defeitos de preparação (bolsões de óleo não rompidos nos processos antecessores à extração). Os meios porosos, segundo Almeida et al. (2010), possuem um conjunto de indicadores, tais como densidade, percolabilidade, porosidade e tortuosidade, que influem nas características da extração. Por exemplo, a alta porosidade provoca densidade baixa, resultando na diminuição da produtividade do extrator. Já a baixa porosidade contribui para a diminuição da percolação, que também diminui a taxa de extração.

A extração do óleo de grãos em escala industrial tem sido praticada desde a década de 1920. Boucher et al. (1942) investigou o processo de extração de óleo impregnado em pratos de argila porosa e constatou que o processo de extração era governado pela lei da difusão molecular. Karnofsky (1949) propôs o modelo da lenta dissolução, que supõe que certos componentes do óleo, como os fosfatídeos, inibem a solubilização dos glicerídeos. Já o modelo do escoamento capilar de Othmer e Agarwal (1955) afirma que a extração consiste em um problema de fluidodinâmica no qual o solvente e o óleo escoam em um complicado sistema formado por células vegetais rompidas com a quebra e a laminação dos grãos. Thomas (2003) estudou o processo de extração de óleos vegetais em um extrator tipo Rotocell e desenvolveu um modelo físico-matemático e um programa para simular a operação do extrator, levando-se em consideração a difusão do óleo pela miscela (óleo + solvente), transferência de óleo entre as fases poro e "bulk", variação da massa específica e viscosidade da miscela.

Um dos trabalhos mais recentes sobre modelagem de extratores de esteira é apresentado por Almeida et al. (2010), abordando um modelo formado por equações diferenciais parciais, que relacionam as concentrações de óleo dentro e fora dos poros do material sólido para todo o campo de extração, e equações algébricas para as concentrações de óleo nas comportas.

No panorama atual, a redução de custos e a otimização desses processos são fatores estratégicos para a sobrevivência das empresas. Segundo Veloso (2003) a análise dos fluxos de transferência de massa em meios porosos tem se destacado nos últimos anos devido às inúmeras aplicações industriais, principalmente com extração contínua por solvente.

Nas plantas de extração de óleo os extratores são equipamentos que precisam ser otimizados e bem operados, para um melhor aproveitamento da matéria prima e estabilidade do processo. Para Almeida et al. (2010) o desenvolvimento de um modelo matemático consiste em um meio de ganhar tempo e evitar perdas no processo, uma vez que a maioria das condições operacionais são estabelecidas empiricamente. Um bom modelo pode ser utilizado para projeto, análise e otimização da operação de extratores. 


\section{O MODELO MATEMÁTICO}

O modelo matemático utilizado no estudo foi proposto por Almeida et al. (2010). Neste modelo o sólido é considerado totalmente poroso, sendo que dentro dos poros o óleo da fase sólida entra em equilíbrio simultâneo com a miscela, e o óleo do filme se transfere para fora dos poros por difusão. Existe, portanto, um gradiente de concentração entre a miscela da fase poro e a miscela da fase "bulk". Um esquema dessa transferência de massa é mostrado na Figura 1. Com a entrada do solvente nos poros ocorre uma transferência simultânea do óleo da fase sólida para a fase poro, mantendo-se um equilíbrio entre essas concentrações e o coeficiente de equilíbrio da extração é definido como $E_{d}^{V}=w / c^{p}$.

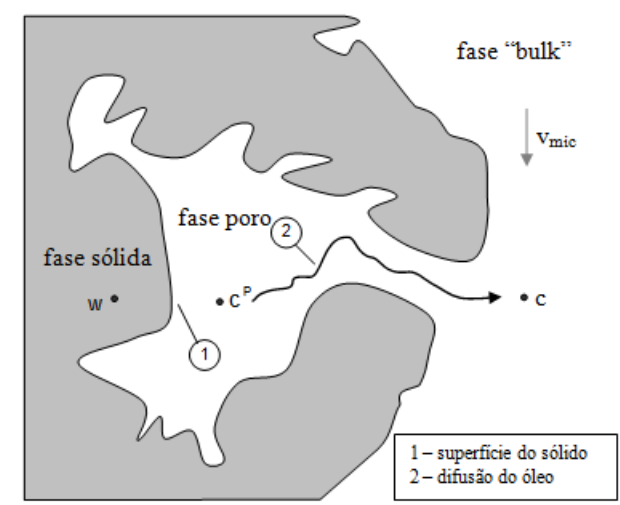

Figura 1 - Transferência de óleo de um poro para a fase "bulk"

O modelo de Almeida et al. (2010) considera dois balanços de massa, um na fase poro e outro na fase "bulk", resultando na seguinte equação diferencial parcial para a fase poro:

$$
-\frac{\partial c^{p}}{\partial t}=\frac{\left(1-\varepsilon^{p}\right)}{\varepsilon^{p}} \frac{\partial w}{\partial t}+\frac{M_{\text {ent }}}{\rho_{\text {poro }} H W} \frac{\partial c^{p}}{\partial x}+\frac{k_{f} a}{\varepsilon^{p}\left(1-\varepsilon^{b}\right)}\left(c^{p}-c\right)
$$

Para a fase "bulk", o modelo apresenta a seguinte equação diferencial parcial:

$$
-\frac{\partial c}{\partial t}=\frac{P}{\varepsilon^{b}} \frac{\partial c}{\partial y}+\frac{M_{\text {ent }}}{\rho_{\text {poro }} H W} \frac{\partial c}{\partial x}-E_{s}\left(\frac{\partial^{2} c}{\partial x^{2}}+\frac{\partial^{2} c}{\partial y^{2}}\right)-\frac{k_{f} a}{\varepsilon^{b}}\left(c^{p}-c\right)
$$

O esquema do leito no interior do extrator de esteira é mostrado na Figura 2.

Para a solução das equações, foram estabelecidas as seguintes condições de contorno:

a) Parte inferior do leito:

$$
\mathrm{c}(\mathrm{x}, 0)=\mathrm{c}_{\mathrm{ent}}, \mathrm{j} \text { e }\left.\frac{\partial \mathrm{w}}{\partial \mathrm{y}}\right|_{\mathrm{y}=0} \quad \text { para } 0<x<L ; i=1 \ldots 10 ; \mathrm{y}=0
$$

b) Parte final do leito:

$$
\left.\frac{\partial \mathrm{c}}{\partial \mathrm{x}}\right|_{\mathrm{x}=\mathrm{L}}=\left.0 \mathrm{e} \frac{\partial \mathrm{w}}{\partial \mathrm{x}}\right|_{\mathrm{x}=\mathrm{L}}=0 \text { para } 0<y<H ; x=L
$$


c) Parte superior do leito:

$$
\left.\frac{\partial \mathrm{c}}{\partial \mathrm{y}}\right|_{\mathrm{y}=\mathrm{H}}=\left.0 \mathrm{e} \frac{\partial \mathrm{w}}{\partial \mathrm{y}}\right|_{\mathrm{y}=\mathrm{H}}=0 \text { para } 0<x<L ; y=\mathrm{H}
$$

d) Parte inicial do leito:

$$
\left.\frac{\partial \mathrm{c}}{\partial \mathrm{x}}\right|_{\mathrm{x}=0}=0 \text { e } \mathrm{w}(0, \mathrm{y})=\mathrm{w}_{\mathrm{ent}} \text { para } 0<y<H ; \mathrm{x}=0
$$

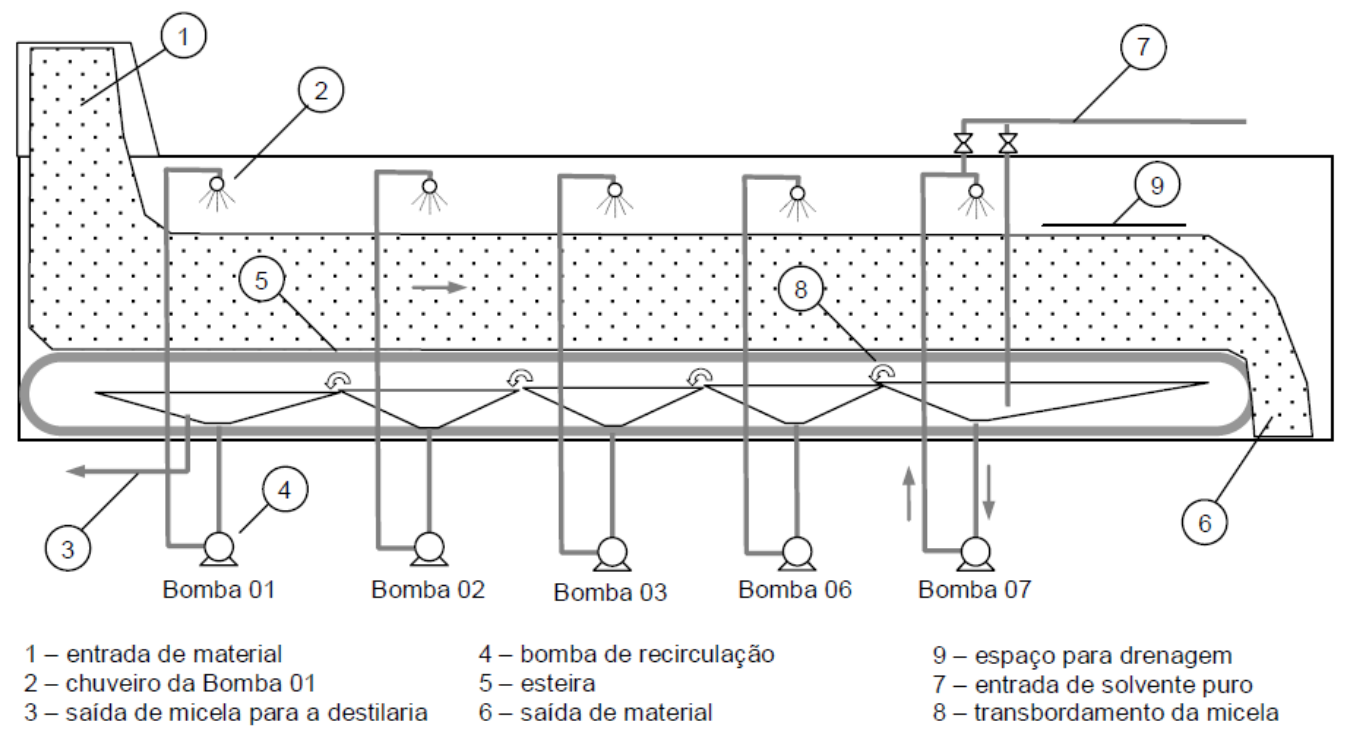

Figura 2 - Esquema de um extrator contínuo tipo esteira

O extrator abordado neste trabalho é similar ao mostrado na Figura 2 e possui 10 bombas de recirculação.

Para resolver o sistema de equações diferenciais parciais desenvolvido para o leito do extrator foi utilizado o método numérico de diferenças finitas. Para isso foram discretizadas as equações diferenciais e o leito representado por uma malha, onde para cada ponto se atribuiu duas equações discretizadas, uma para a concentração de óleo na miscela (c) e outra para a concentração de óleo no sólido (w), baseadas nas condições de contorno, conforme mostra a Figura 3(a). Montou-se um sistema de equações algébricas e acoplou-se os dois sistemas gerados em um único sistema linear para solução do problema.

A Figura 3(a) é composta por $m$ pontos horizontais e $n$ pontos verticais, totalizando $m . n$ nós em toda a malha. Cada nó representa um ponto para o qual serão calculados os valores das concentrações de óleo na miscela (c) e no sólido (w). Como o leito possui comprimento L e altura $\mathrm{H}$, as distâncias entre cada nó serão $\Delta \mathrm{x}=\mathrm{L} /(\mathrm{m}-1)$ e $\Delta \mathrm{y}=\mathrm{H} /(\mathrm{n}-1)$, na horizontal e vertical, respectivamente. A Figura 3(b) mostra as concentrações em cada nó em torno do nó $(\mathrm{i}, \mathrm{j})$. 


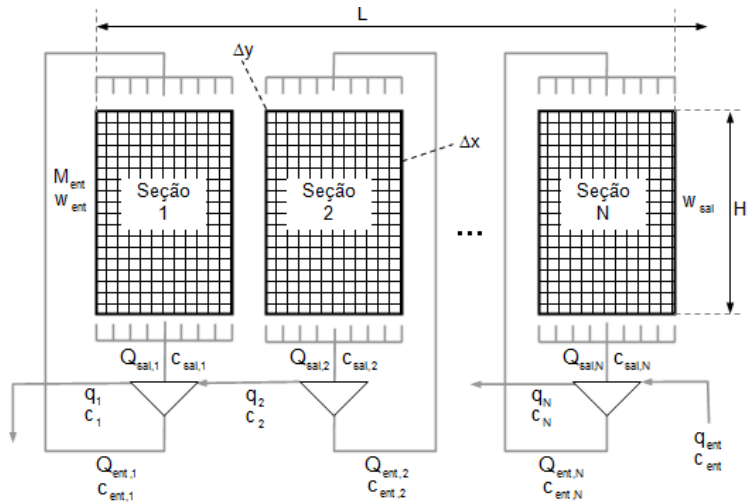

(a)

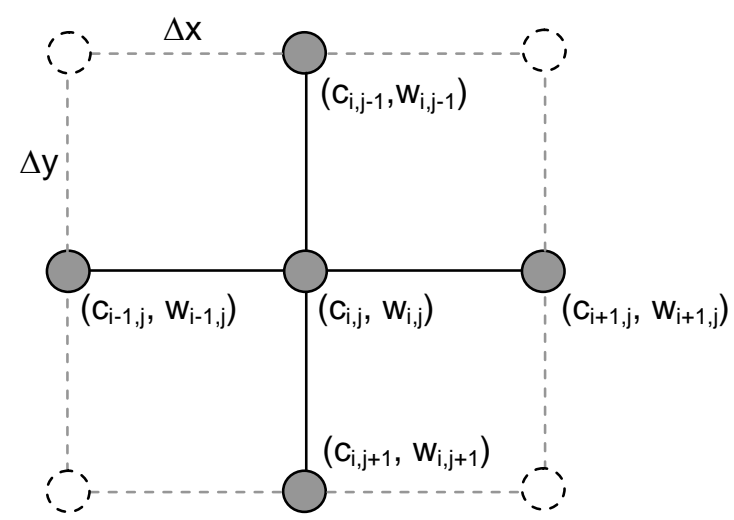

(b)

Figura 3 - Representação da malha e das concentrações em cada nó

\section{RESULTADOS}

O modelo de Almeida et al. (2010) foi ajustado, por meio da minimização de uma função objetivo (Equação 7), de forma a representar o fenômeno físico do processo de extração em um extrator de esteira com recirculação de miscela. Esta função, denominada $\varphi$, leva em consideração as concentrações das miscelas em cada comporta e também o teor residual de óleo no farelo, na saída do extrator.

$$
\varphi=\frac{1}{10} \sum_{i=1}^{10}\left(\frac{C_{i}^{\text {exp }}-C_{i}^{\text {modelo }}}{C_{i}^{\text {exp }}}\right)^{2}+\left(\frac{w^{\text {exp }}-w^{\text {modelo }}}{w^{\text {exp }}}\right)^{2}
$$

A partir da Equação 7 e da aplicação do modelo de Almeida et al. (2010) foi possível determinar o valor do coeficiente de equilíbrio da extração para quatro condições distintas de operação do extrator: concentração de óleo na miscela na saída do extrator de $30,14 \%$, $27,57 \%, 24,03 \%$ e $21,01 \%$.

A Tabela 2 apresenta os parâmetros fixos utilizados para as quatro condições estudadas.

Tabela 2 - Parâmetros e dados do extrator, meio poroso, óleo e solvente

\begin{tabular}{|c|c|c|c|c|}
\hline $\mathrm{L}(\mathrm{m})$ & $\mathrm{H}(\mathrm{m})$ & $\mathrm{W}(\mathrm{m})$ & $\rho_{\text {sol }}\left(\mathrm{kg} / \mathrm{m}^{3}\right)$ & $\mathrm{P}\left(\mathrm{m}^{3} / \mathrm{m}^{2} . \mathrm{h}\right)$ \\
\hline 12,00 & 2,00 & 1,30 & 520 & 60 \\
\hline$\varepsilon^{\mathrm{b}}$ & $\varepsilon^{\mathrm{p}}$ & $\mathrm{a}\left(\mathrm{m}^{2} / \mathrm{m}^{3}\right)$ & $\mathrm{k}_{\mathrm{f}}(\mathrm{m} / \mathrm{s})$ & $\mathrm{E}_{\mathrm{s}}(\mathrm{m} / \mathrm{s})$ \\
\hline 0,40 & 0,30 & 1.650 .000 & $3,2.10^{-9}$ & $1,3.10^{-6}$ \\
\hline $\mathrm{Q}_{\text {ent, }, \mathrm{a}} \mathrm{Q}_{\text {ent, }, 5}\left(\mathrm{~m}^{3} / \mathrm{h}\right)$ & $\mathrm{Q}_{\text {ent, }, 9}\left(\mathrm{~m}^{3} / \mathrm{h}\right)$ & $\mathrm{Q}_{\text {ent, }, 6} \mathrm{a} \mathrm{Q}_{\text {ent, }, 10}\left(\mathrm{~m}^{3} / \mathrm{h}\right)$ & $\mathrm{m}$ & $\mathrm{n}$ \\
\hline 72 & 60 & 50 & 41 & 10 \\
\hline
\end{tabular}

Alguns parâmetros utilizados no estudo variaram de acordo com a condição de operação, conforme mostra a Tabela 3 . Em cada condição foi utilizada uma malha não uniforme para discretização do leito, com $\Delta x=0,30$ e $\Delta y=0,21$.

Para a solução do modelo aplicado a cada condição distinta de operação, levando-se em consideração os dados das Tabelas 2 e 3, utilizou-se o sistema algébrico computacional Maple ${ }^{\circledR}$ versão 13, produzido e distribuído pela empresa canadense Waterloo Maple Inc, e os 
Tabela 3 - Parâmetros operacionais e da matéria prima

\begin{tabular}{|l|c|c|c|c|c|c|c|}
\hline & $\begin{array}{c}\text { Concentraçã } \\
\text { da miscela na } \\
\text { saída do } \\
\text { extrator }\end{array}$ & $\mathrm{c}\left(\mathrm{kg} / \mathrm{m}^{3}\right)$ & $\mathrm{T}\left({ }^{\circ} \mathrm{C}\right)$ & $\mathrm{E}_{\mathrm{d}}^{\mathrm{v}}$ & $\mathrm{M}_{\mathrm{ent}}(\mathrm{kg} / \mathrm{h})$ & $\begin{array}{c}\mathrm{w}_{\text {ent }} \\
\left(\mathrm{kg} / \mathrm{m}^{3}\right)\end{array}$ & $\mathrm{q}_{1}\left(\mathrm{~m}^{3} / \mathrm{h}\right)$ \\
\hline Condição 1 & $30,14 \%$ & 219,85 & 56 & 0,131 & 24.000 & 105,25 & 26 \\
\hline Condição 2 & $27.57 \%$ & 201,01 & 56 & 0,140 & 25.000 & 104,05 & 26 \\
\hline Condição 3 & $24,03 \%$ & 175,28 & 56 & 0,169 & 22.000 & 103,17 & 24 \\
\hline Condição 4 & $21,01 \%$ & 153,25 & 56 & 0,185 & 25.000 & 105,05 & 26 \\
\hline
\end{tabular}

resultados obtidos para as concentrações de óleo na miscela das bombas do extrator e de óleo na fase sólida ao longo do comprimento do extrator são mostrados nas Figura 4 e 5.
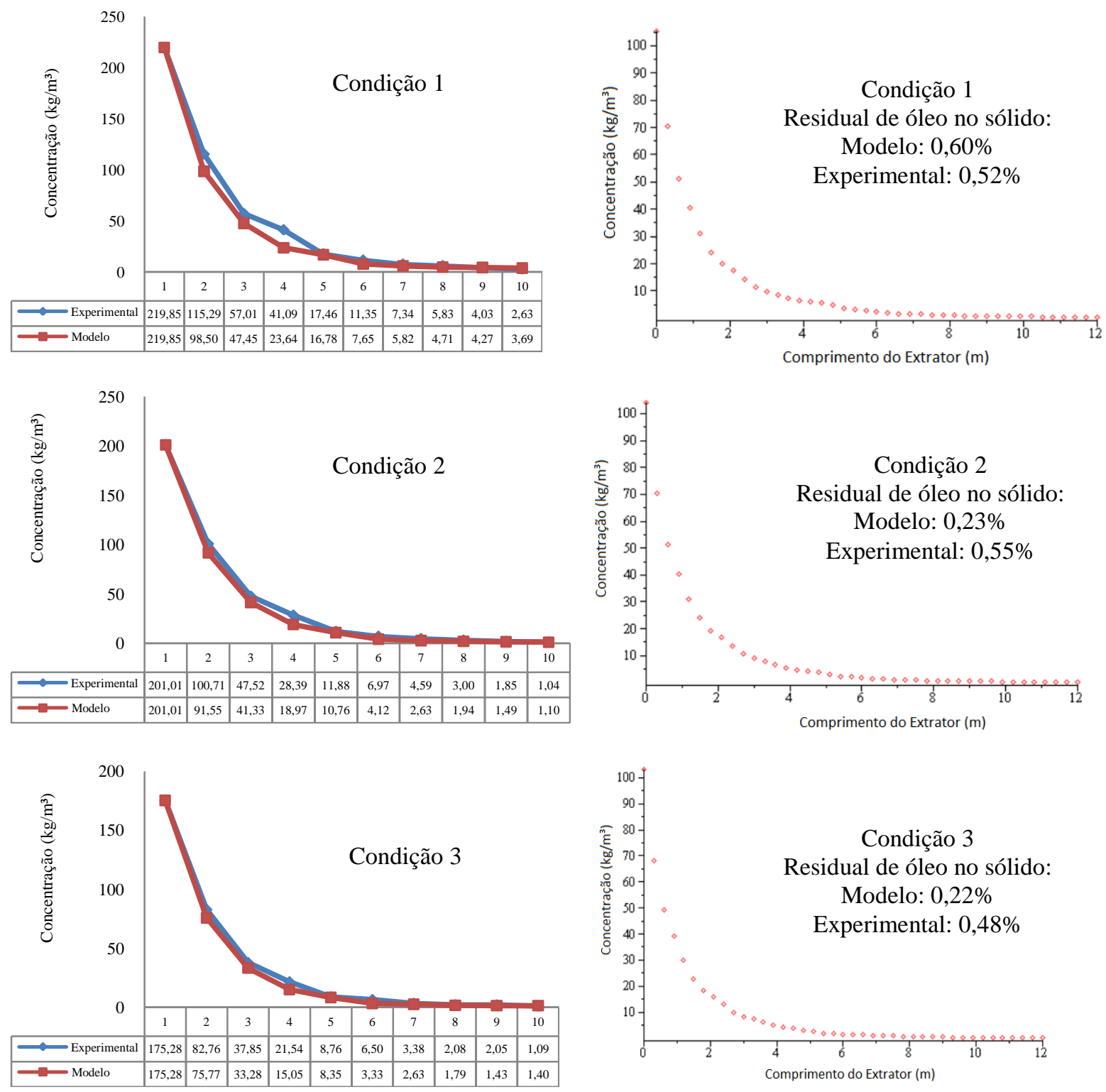

Figura 4 - Concentração de óleo nas bombas de miscela e na fase sólida ao longo do extrator 

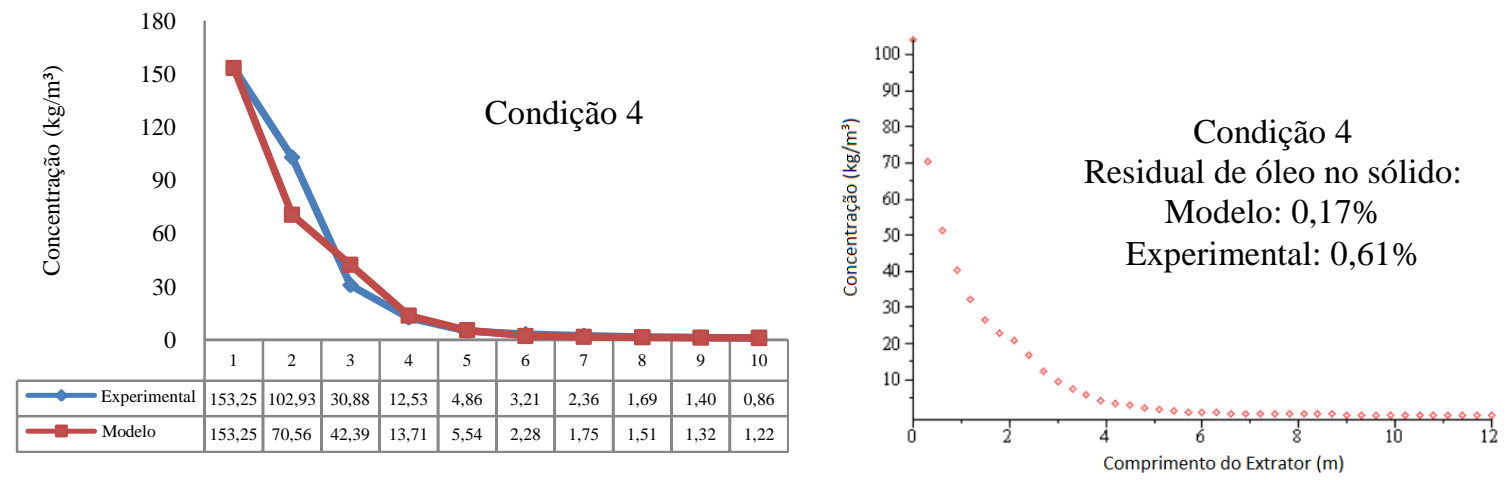

Figura 5 - Concentração de óleo nas bombas de miscela e na fase sólida ao longo do extrator

Para o processo industrial estudado, com a aplicação do modelo proposto por Almeida et al. (2010), foi possível determinar os coeficientes de equilíbrio da extração para cada condição distinta de operação do extrator de esteira, variando a concentração de óleo na miscela, na saída do equipamento. A Figura 6 mostra a variação do coeficiente de equilíbrio da extração com a variação da concentração da miscela na saída do extrator.

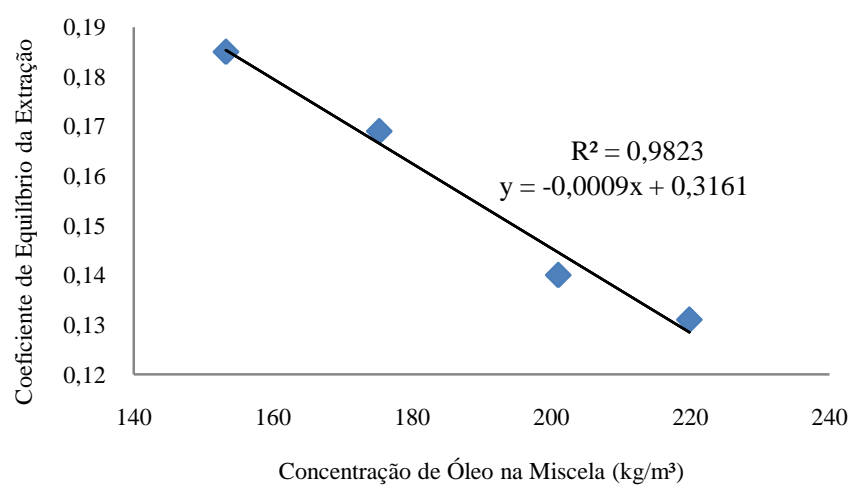

Figura 6 - Variação do coeficiente de equilíbrio da extração em função da concentração de óleo na miscela na saída do extrator

\section{CONCLUSÕES}

Os resultados obtidos a partir da aplicação do modelo proposto por Almeida et al. (2010) a um extrator industrial De Smet de esteira com recirculação de miscela apresentaram baixos desvios em relação aos valores experimentais do processo, mostrando que o modelo pode ser aplicado na simulação, análise e otimização de extratores industriais de óleo de soja.

Em seu estudo de caso, Almeida et al. (2010) atribuiu os desvios a diversos fatos, sendo um deles a relação de equilíbrio, a qual alega que diversos modelos da literatura adotam como sendo linear, mas que carece de melhores estudos. A aplicação do modelo a um processo industrial mostrou que, para o extrator estudado, esta variação realmente é linear, com um coeficiente de determinação $\mathrm{R}^{2}$ de 0,9823 , confirmando a informação dos demais modelos quanto à linearidade da relação de equilíbrio com relação à concentração. 


\section{NOMENCLATURA}

a - área de contato entre as fases poro e "bulk" $\left(\mathrm{m}^{2} / \mathrm{m}^{3}\right)$

c - concentração de óleo na fase "bulk" $\left(\mathrm{kg} / \mathrm{m}^{3}\right)$

$c^{p}-$ concentração de óleo na fase poro $\left(\mathrm{kg} / \mathrm{m}^{3}\right)$

$\mathrm{E}_{\mathrm{s}}$ - coeficiente de dispersão $\left(\mathrm{m}^{2} / \mathrm{s}\right)$

$\mathrm{E}_{\mathrm{d}}^{\mathrm{V}}$ - coeficiente volumétrico de equilíbrio do óleo entre as fases sólido e poro (adimensional)

$\mathrm{H}$ - altura do estágio ou do leito do extrator (m) $\mathrm{k}_{\mathrm{f}}-$ coefic. efetivo de transferência de massa $(\mathrm{m} / \mathrm{s})$

$\mathrm{L}$ - comprimento da zona de extração (m)

$\mathrm{M}_{\mathrm{ent}}$ - fluxo mássico de sólidos $(\mathrm{kg} / \mathrm{h})$

$\mathrm{m}$ e $\mathrm{n}-\mathrm{n}^{\circ}$ de pontos horizontais e verticais, respectivamente q - vazão volum. miscela nas comportas

$\left(\mathrm{m}^{3} / \mathrm{h}\right)$

$\mathrm{Q}$ - vazão volumétrica de miscela $\left(\mathrm{m}^{3} / \mathrm{h}\right)$

$\mathrm{t}$ - tempo (s)

$\mathrm{V}_{\text {mic }}$ - velocidade de percolação $(\mathrm{m} / \mathrm{s})$

$\mathrm{v}_{\text {est }}$ - velocidade da esteira $(\mathrm{m} / \mathrm{s})$

$\mathrm{W}$ - largura do estágio ou leito (m)

w - concentração óleo fase sólida $\left(\mathrm{kg} / \mathrm{m}^{3}\right)$

$\mathrm{x}$ e $\mathrm{y}$ - coordenadas espaciais do leito

$\varepsilon^{\mathrm{p}}$ - porosidade externa (adimensional)

$\varepsilon^{\mathrm{b}}$ - porosidade interna (adimensional)

$\rho_{\mathrm{sol}}-$ massa espec. fase sólida $\left(\mathrm{kg} / \mathrm{m}^{3}\right)$

$\rho_{\text {poro }}-$ massa espec. meio poroso $\left(\mathrm{kg} / \mathrm{m}^{3}\right)$

\section{REFERÊNCIAS}

ALMEIDA, R. L.; MODENES, A. N.; RAVAGNANI, M. A. S.S. Soybean oil extraction in belt extractors with miscella recirculation. Chemical Engineering and Processing: Process Intensification, v. 49, Elsevier B. V., 2010.

BOUCHER, D. F., BRIER, J. C., OSBORN, J. O., 1942. Extraction of soybean. Transfer American Institute Chemical Engineering, v. 38.

BOSS, E. A. Análise do Desempenho de Plantas de Extração de Óleo Convencionais e de Processos Supercríticos. 2000. Dissertação (Mestrado) - Faculdade de Engenharia, Universidade Estadual de Campinas, Campinas.

DEMARCO, A. Extracción por solvente em Temas Selectos em Aceites y Grasas. BLOCK, J. M.; BARREIRA-ARELLANO, D. Eds., São Paulo: Blucher, 2009.

KARNOFSKY, G., 1949. The Theory of Solvent Extraction. JAOCS - Journal of American Oil Chemistry Society, v. 26

OTHMER, D. F., AGARWAL, J. C., 1955. Extraction of Soybeans - Theory and Mechanism. Chemical Engineering Progress, v. 51, n. 8 (ago).

RATAUS, M. La Extracción por Solvente. In: A\&G Libro $10^{\circ}$ Aniversario. Recopilaciónde Artículos Técnicos - Ediciones 1 a 41 - 1990 a 2000. Asociacion Argentina de Grasas Y Aceites.

THOMAS, G. C. Análise Teórico-Experimental da Extração de Óleo de Soja em Instalação Industrial do Tipo Rotocell. 2003. Tese (Doutorado), Porto Alegre, 2003.

VELOSO, G. O. Modelagem Física e Matemática dos Processos de Extração de Óleo de Soja em Fluxos Contracorrente Cruzados. 2003. Tese (Doutorado), Porto Alegre, 2003. 